\title{
Diversity in boron toxicity tolerance of Australian barley (Hordeum vulgare L.) genotypes
}

\author{
Julie E. Hayes ${ }^{1 *}$ (D) Margaret Pallotta ${ }^{1}$, Melissa Garcia ${ }^{1}$, Mehmet Tufan Öz ${ }^{1,2}$, Jay Rongala ${ }^{1}$ and Tim Sutton ${ }^{1,3}$
}

\begin{abstract}
Background: Boron (B) is an important micronutrient for plant growth, but is toxic when levels are too high. This commonly occurs in environments with alkaline soils and relatively low rainfall, including many of the cereal growing regions of southern Australia. Four major genetic loci controlling tolerance to high soil B have been identified in the landrace barley, Sahara 3771. Genes underlying two of the loci encode the B transporters HvBot1 and HVNIP2;1.
\end{abstract}

Results: We investigated sequence and expression level diversity in HvBot1 and HVNIP2;1 across barley germplasm, and identified five novel coding sequence alleles for HvBot1. Lines were identified containing either single or multiple copies of the Sahara HvBot1 allele. We established that only the tandemly duplicated Sahara allele conferred B tolerance, and this duplicated allele was found only in a set of nine lines accessioned in Australian collections as Sahara 3763-3771. HvNIP2;1 coding sequences were highly conserved across barley germplasm. We identified the likely causative SNP in the $5^{\prime}$ UTR of Sahara HVNIP2;1, and propose that the creation of a small upstream open reading frame interferes with HvNIP2;1 translation in Sahara 3771. Similar to HvBot1, the tolerant HvNIP2; 1 allele was unique to the Sahara barley accessions. We identified a new source of the $2 \mathrm{H} \mathrm{B}$ tolerance allele controlling leaf symptom development, in the landrace Ethiopia 756.

Conclusions: Ethiopia 756, as well as the cultivar Sloop Vic which carries both the $2 \mathrm{H}$ and HvBot1 B tolerance alleles derived from Sahara 3771, may be valuable as alternative parents in breeding programs targeted to high soil $\mathrm{B}$ environments. There is significant diversity in B toxicity tolerance among contemporary Australian barley varieties but this is not related to variation at any of the four known B tolerance loci, indicating that novel, as yet undiscovered, sources of tolerance exist.

\section{Background}

High soil boron (B) can affect yields of barley (Hordeum vulgare L.) across southern Australia by up to $17 \%$ [1], depending on a multitude of site, seasonal and genetic factors [2-5]. Along with disease ratings, standard information for new barley varieties released in South Australia, Victoria and Western Australia often includes a boron tolerance rating [6, 7], allowing farmers to select varieties tolerant to high soil B. Genetic variability for high $\mathrm{B}$ tolerance has long been known $[8,9]$. The most tolerant barley identified amongst breeding material in Australia is the unadapted six-row North African landrace, Sahara 3771. This genotype was accessioned in

\footnotetext{
*Correspondence: julie.hayes@acpfg.com.au

${ }^{1}$ Australian Centre for Plant Functional Genomics and The University of Adelaide, Adelaide, South Australia, Australia

Full list of author information is available at the end of the article
}

Australian collections in the early 1900s [10], one of a set of nine barley lines listed as Sahara 3763 - Sahara 3771. It has been considered an important source of $\mathrm{B}$ tolerance for barley breeding programs over many years. Four major QTL for B tolerance were identified in Sahara 3771, in a genetic study using a doubled haploid (DH) population derived from a cross between the South Australian malting variety, Clipper, and Sahara 3771 [11]. Subsequent research to finemap two of the regions revealed the identity of the tolerance genes HvBot1 (chromosome 4H) [12] and HvNIP2;1 (chromosome 6H) [13]. They encode two types of transporter that function to minimise the amount of B in barley roots. These genes have been partially characterised, but the prevalence of the tolerant alleles across Australian germplasm was not known. It was also not known if there is significant 
diversity in $H v B o t 1$ and $H v N I P 2 ; 1$ contributing to B tolerance, such as has been found in wheat for TaBot-B5 [14]. Therefore, the aims of this study were to: 1) determine the prevalence of known $B$ tolerance alleles in Australian barley germplasm; 2) develop an improved set of markers for tracking the introgression of B tolerance from Sahara 3771; and 3) identify alternative sources of B tolerance in barley. This was a broad study and, although the set of germplasm assessed was not exhaustive, our data suggest that the tolerance alleles found in Sahara 3771 are rare. The significance of a QTL on chromosome $2 \mathrm{H}$ controlling leaf symptom expression is highlighted as a target for future breeding and selection for B tolerance in barley.

\section{Results}

Tolerance to high soil B in the barley landrace Sahara 3771 has been attributed to four major QTL, on chromosomes $2 \mathrm{H}, 3 \mathrm{H}, 4 \mathrm{H}$ and $6 \mathrm{H}$. We screened a set of 65 diverse barley genotypes (Additional file 1: Table S1) for variation at these loci using genomic Southern analysis, which also enabled us to assess gene copy number variation. Coding sequence for the genes encoding $B$ transport proteins HvBot1 and HvNIP2;1, and which lie beneath the $4 \mathrm{H}$ and $6 \mathrm{H}$ tolerance loci, respectively, was also amplified and sequenced. In sourcing diverse germplasm to screen, we obtained seed for nine barleys accessioned in the Australian Grains Genebank as Sahara 3763 to Sahara 3771. Our analyses suggest that the Sahara accessions possess a unique set of $\mathrm{B}$ tolerance alleles.

\section{Genetic variation at the $4 \mathrm{H}$ locus (HvBot1)}

Southern analysis of the 65 barley genotypes revealed that the tandem HvBot1 gene duplication found in Sahara 3771 is rare, although other genotypes (eg. California Mariout and derivative cultivars CM67 and CM72, and the Japanese cultivars Haruna Nijo and Amagi Nijo) showed a Saharalike restriction pattern without gene duplication (panel A, Additional file 2: Figure S1). All nine Sahara genotypes from the Australian Grains Genebank possessed the $H v B o t 1$ gene duplication, and displayed similarly high B-tolerant phenotypes in hydroponic experiments (Additional file 2: Figure S1).

Sequencing of the coding regions of $H v B o t 1$ revealed seven coding sequence variants (Fig. 1a), including the Clipper and Sahara (single- and multi-copy) HvBot1 alleles. Eight synonymous SNPs were found between the Clipper and Sahara alleles, with no resulting amino acid changes ([GenBank:EF660437], Clipper HvBot1 complete CDS, has been updated to reflect this finding). Amino acid substitutions were found in each of the other five variants, at either one (Tadmor, Alexis and Morex) or two (Haruna Nijo and WI4304) of five different locations. The
Alexis, Morex and WI4304 variants shared a common substitution ( $\left.\mathrm{Asn}^{108} \mathrm{Ser}\right)$. We have named the allelic variants Bot1.a through to Bot1.g, as illustrated in Fig. 1a. Coding sequences for the novel alleles have been lodged with GenBank [GenBank:KR605456, GenBank: KR605457, GenBank:KR605458, GenBank:KR605459, Gen Bank:KR605460].

We compared HvBot1 expression levels in roots of seedlings of barley genotypes carrying the different alleles (Fig. 1b). HvBot1 expression was similar for all variants except for genotypes carrying the duplicated Sahara allele $(\operatorname{Bot} 1(D p) \cdot b)$, which in this experiment showed more than 200-fold higher levels of expression (Fig. 1b). Multiple sequencing efforts failed to uncover any sequence differences, in either the HvBot1 gene or its promoter, between copies in lines carrying the HvBot1 duplication, or between the duplicated allele and lines containing a single copy of the Sahara HvBot1 allele.

We identified an individual $F_{2}$ seedling from a cross between two Clipper X Sahara 3771 DH lines, which carried a chromosome with a recombination break point between the HvBot1 gene copies and a chromosome with an intact Sahara 3771 gene cluster. Southern analysis identified homozygous recombinant progeny which likely retained only one of the copies of this gene. No sequence differences were identified between the HvBot1 copy in the recombinant and those in the Sahara parent. We used this recombinant line to further investigate the impact of $H v B o t 1$ duplication on expression. Levels of HvBot1 expression in roots of homozygous recombinant $F_{3}$ progeny were similar to expression levels in Clipper, while progeny with the full set of duplicated HvBot1 copies showed similar levels of HvBot1 expression to Sahara, and the heterozygous progeny had intermediate expression (Fig. 2).

We also assessed the function of each of the variants by heterologous expression in yeast (Saccharomyces cereviseae). With the exception of the Haruna Nijo allele (Bot1.d), all variants conferred a similar level of tolerance to media containing high concentrations of $B$ when expressed in yeast (Panel A in Additional file 3: Figure S2). Clones containing the Haruna Nijo variant were no more tolerant to high B than empty vector control yeast, indicating that one or both of the residue substitutions in the Haruna Nijo HvBot1 allele disrupted protein function. We mutated each of the residues separately in the Sahara HvBot1 ORF and tested the two mutations in a second yeast expression experiment, which demonstrated that Leucine at position 234 in HvBot1 is critical for function (Fig. 1c). By contrast, the Thr541Met substitution had no effect on transporter function. The cultivars Haruna Nijo and Amagi Nijo which carry the Haruna Nijo HvBot1 allele are intolerant to high $B$ when grown in hydroponics. Root B concentrations were similar to 
(a)

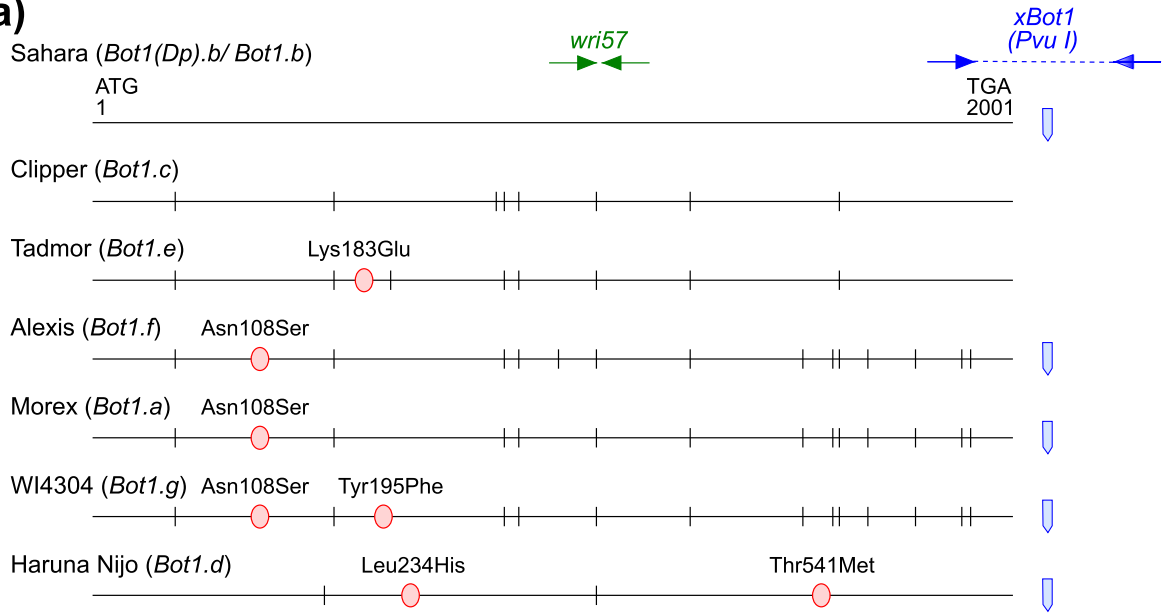

(b)

(c)
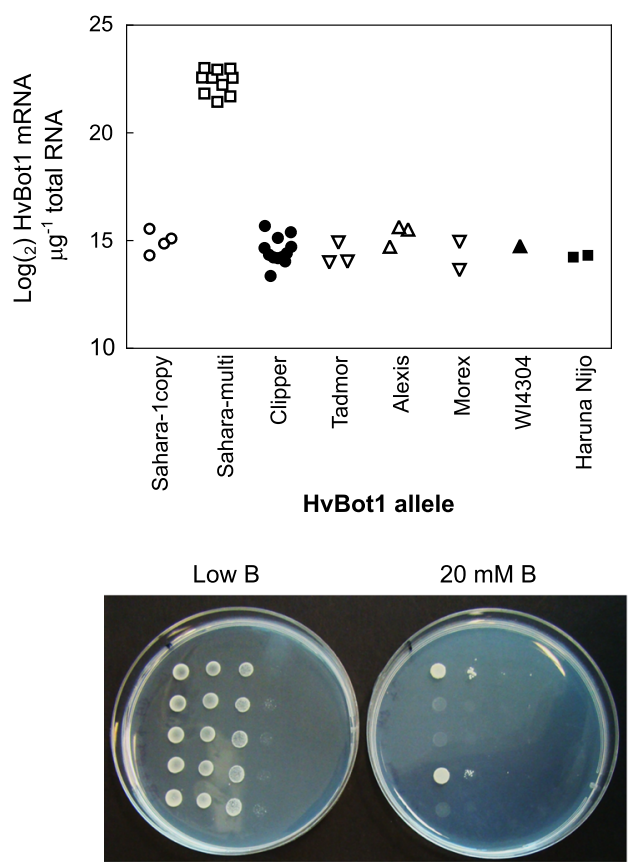

Bot1.b

Bot1.d

Bot1.b (Leu234His)

Bot1.b (Thr541Met)

empty vector

Fig. 1 Genetic variation in barley at the $4 \mathrm{H}$ B tolerance locus (HvBot1). a HvBot1 coding sequence alleles detected in a diverse set of barley genotypes. All SNPs relative to the Sahara allele are shown as lines, with those resulting in an amino acid change shown as circles. The positions of the XBot1 CAPS marker (blue) and the Sahara-specific wri57 KASP TM assay (green) are also indicated. Allele nomenclature (Bot1.a to Bot1.g) is given according to published guidelines for barley [35]. We designated the allele from the sequencing reference genome of cv. Morex as Bot 1.a. b Expression levels of HvBot1 determined by q-RT-PCR, in roots of seedlings of barley genotypes carrying different HvBot1 alleles. Refer to Additional file 8: Table S5 for a list of barley genotypes included in each HvBot1 allele class. Transcript levels were normalised using the RNA housekeeping genes for tubulin, GAPDH and HSP70. c Growth of yeast expressing the Sahara HvBot1 allele (Bot1.b), the non-functional Haruna Nijo HvBot1 allele containing two residue substitutions relative to Sahara (Bot1.g; Leu234His/Thr541Met), and Bot1.b with single residue mutations (Leu234His or Thr541Met) relative to an empty vector control, on a low B medium (left) or at high B (right). Clones are positioned vertically and spots across the plates represent $10 \mathrm{~L}$ of $10^{-0}, 10^{-1}, 10^{-2}$ and $10^{-3}$ dilutions from a starting culture containing approximately $3 \times 10^{7}$ cells $\mathrm{L}^{-1}$. Photographs were taken after $2 \mathrm{~d}$ (low $B$ ) or $4 \mathrm{~d}$ $(20 \mathrm{mM} \mathrm{B})$ incubation at $30^{\circ} \mathrm{C}$, and are representative of two experiments using independent clones

Clipper, and suggested that no net efflux of B from the roots was occurring in either cultivar (Panel B in Additional file 3: Figure S2).

We developed a $\mathrm{KASP}^{\mathrm{TM}}$ marker to track a SNP in exon 11 unique to Sahara (wri57; Additional file 4: Table S2, with marker location shown in Fig. 1a). The previously reported $x$ Bot1 marker [12] (location shown in Fig. 1a) was designed around a SNP that is not specific to the Sahara HvBot1 allele, but is also common to the Alexis, Morex, WI4304 and Haruna Nijo alleles (Fig. 1a). 


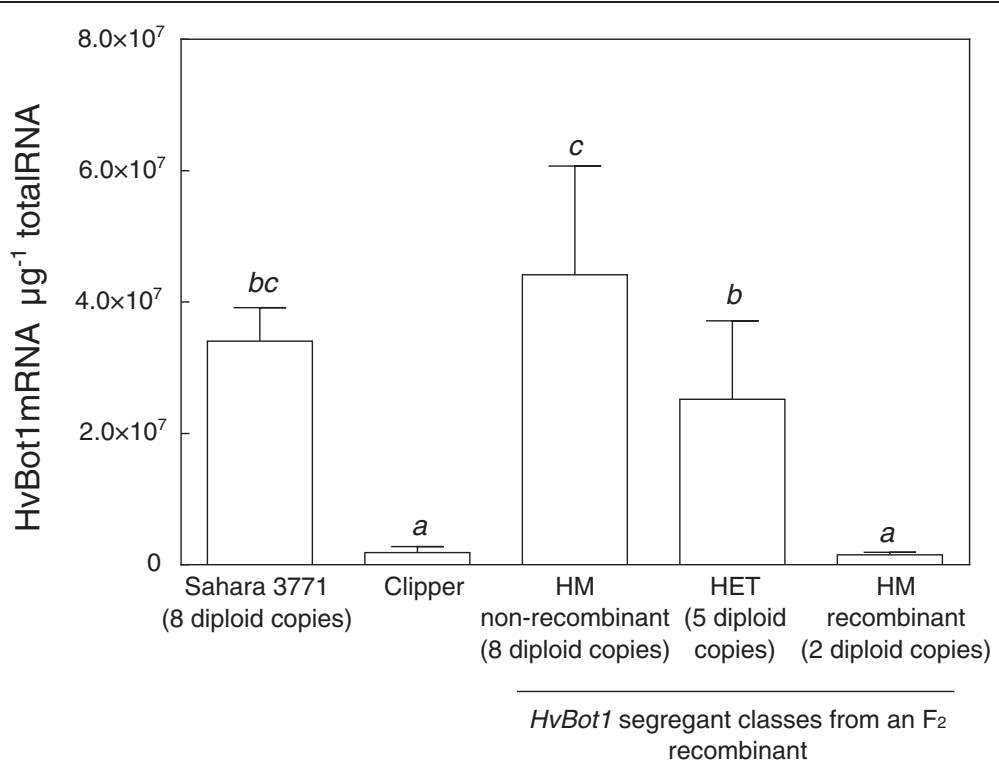

Fig. 2 HvBot1 duplication is correlated with higher levels of HvBot1 expression. HvBot1 transcript levels $(N=6, \pm s d)$ in barley roots of the parental genotypes Sahara 3771 and Clipper, and in HvBot1 segregant classes from an $F_{2}$ recombinant derived from a cross between two doubled haploid lines. Italicised letters above the bars denote significant differences between genotypes (Tukey's multiple comparisons test; $P=0.05$ ). The $F_{2}$ was identified as recombinant within the HvBot1 gene cluster, resulting in $F_{3}$ progeny with different numbers of copies of the Sahara HvBot1 allele. Southern analysis indicated that the homozygous recombinant progeny contained only a single copy of the Sahara HvBot1 allele. Seedlings were grown for 14 days in nutrient solution with an additional $2 \mathrm{mM} \mathrm{B} \mathrm{prior} \mathrm{to} \mathrm{sampling.} F_{3}$ individuals were genotyped using the closely linked XBM178 CAPS marker (S3 Table), to identify segregant classes

\section{Genetic variation at the $6 \mathrm{H}$ locus (HvNIP2;1)}

Initial Southern analysis of barley germplasm using a probe designed against $H v N I P 2 ; 1$ identified three patterns of hybridisation. Sequencing of the coding regions of HvNIP2;1 indicated that the HvNIP2;1 ORF is very highly conserved; a single synonymous SNP occurs between the alleles from Clipper and Sahara 3771 (Fig. 3a). The Clipper sequence variant was found in just over half of all germplasm included for sequencing. Expression levels for $H v N I P 2 ; 1$ in barley roots did not differ either between the two sequence variants or between the three allelic groups identified by genomic Southern analysis (Fig. 3b).

A second SNP, unique to the Sahara accessions and located 44 bp upstream of the ATG start codon in the 5' UTR, was identified in $H v N I P 2 ; 1$. At this position the thymidine base in Sahara creates a short, upstream ORF (uORF) encoding a translatable peptide of thirteen residues immediately upstream of, and in frame with the HvNIP2;1 ORF (Fig. 3a). We hypothesised that this uORF in Sahara may interfere with HvNIP2;1 translation, and be the principal mechanism for reduced function of HvNIP2;1 leading to reduced B permeability and a greater tolerance to high $\mathrm{B}$. The $133 \mathrm{bp} 5$ 'UTR sequences from Clipper and Sahara HvNIP2;1 were cloned into the SP6 vector, immediately upstream of the firefly (Photinus pyralis) luciferase gene driven by the SP6 RNA polymerase promoter. In vitro transcription/translation reactions with each construct demonstrated that the Sahara 5' UTR sequence inhibited translation of the luciferase reporter gene by $43 \%$ relative to the Clipper 5' UTR sequence (Fig. 3c).

Interestingly, the uORF SNP was found only in Sahara 3771 and the other eight Sahara accessions, suggesting that tolerance to high soil B attributable to this locus is very rare. We developed a $\mathrm{KASP}^{\mathrm{TM}}$ marker to track the Sahara uORF SNP in HvNIP2;1 (wri59; Additional file 4: Table S2).

\section{Genetic variation at the $3 \mathrm{H}$ and $2 \mathrm{H}$ loci}

The genetic determinants at these loci are unknown. However, a member of the Bot B transporter gene family, $H v B o t 2$, is located within the region of the $3 \mathrm{H} \mathrm{QTL}$ controlling relative root length at high $\mathrm{B}$ and is considered to be a likely candidate gene [15] ([GenBank:KR817581] lists the coding sequence for HvBot2 from Clipper). The $H v B o t 2$ sequence in Sahara 3771 is disrupted by a large deletion (approx. $5.1 \mathrm{kB}$ ) beginning at the 3' end of the CDS, which may result in a loss of function. We designed two sets of primers, to amplify within and across the deletion, respectively (Additional file 5: Table S3). Discriminating PCR determined that with the exception of the nine Sahara accessions, the HvBot2 CDS was intact in all tested barley genotypes. A KASP ${ }^{\mathrm{TM}}$ marker was developed to track the Sahara 3771 deletion (wri58; Additional file 4: Table S2). 


\section{a}

Clipper

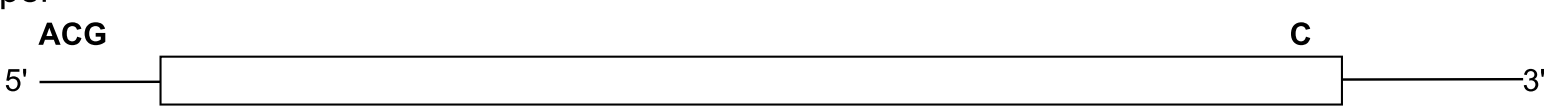

Sahara

ATG

G
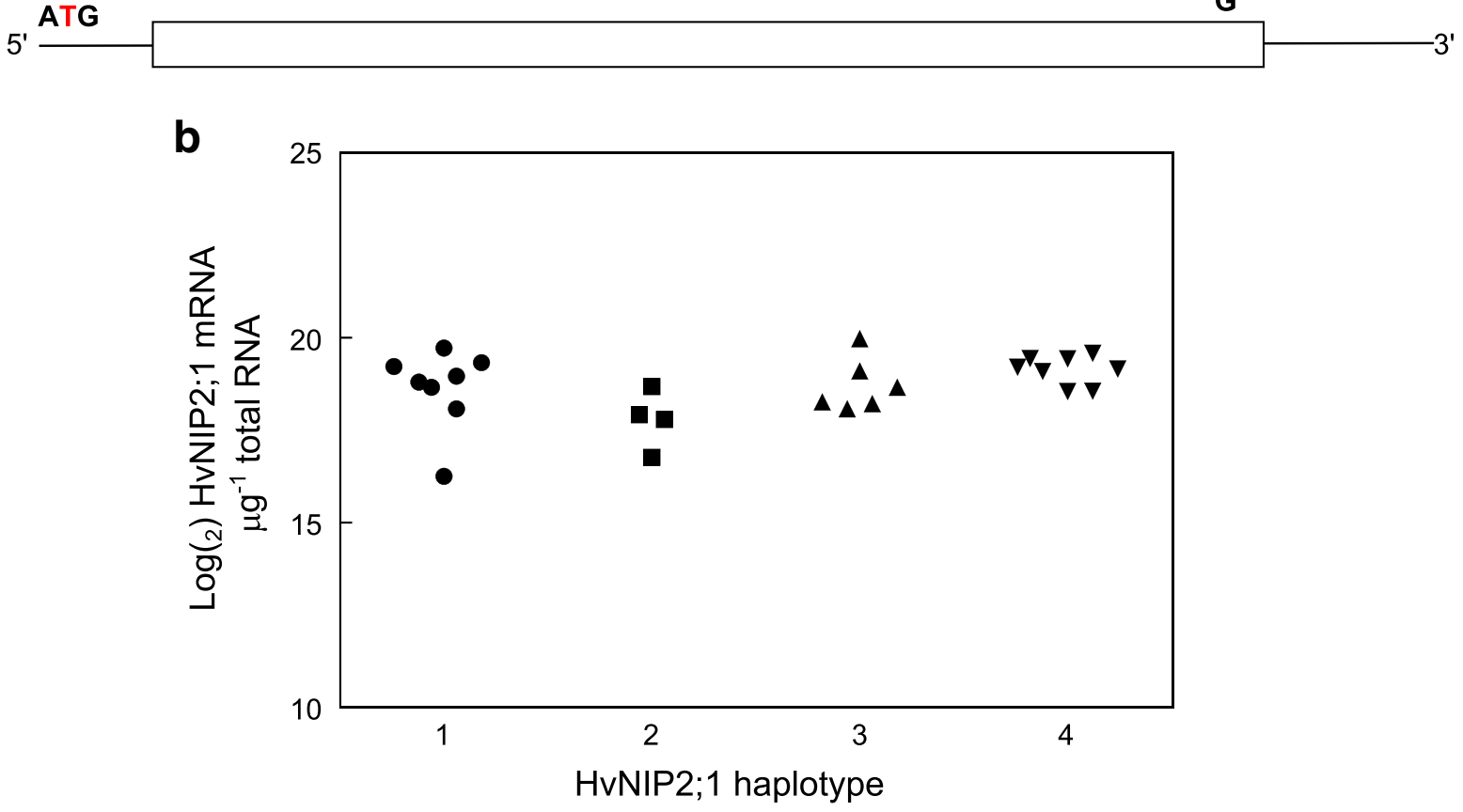

C

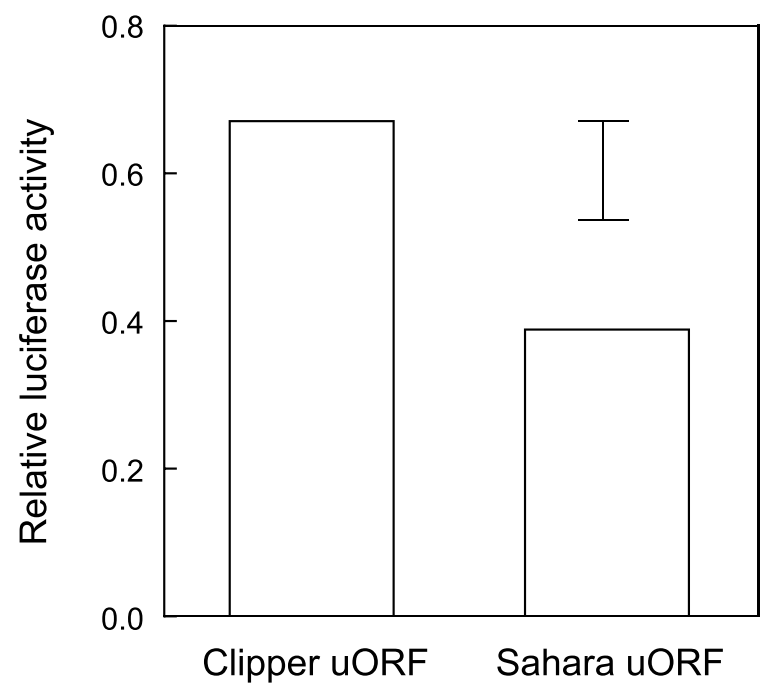

Fig. 3 (See legend on next page.) 
(See figure on previous page.)

Fig. 3 Genetic variation in barley at the 6H B tolerance locus (HVNIP2;1). a Schematic of HvNIP2;1 coding sequences from Clipper and Sahara showing positions of two identified SNPs. No other sequence variation was found among 68 barley genotypes. A KASPTM marker assay was designed against the Sahara-specific 5'UTR SNP. $\mathbf{b}$ Expression levels of HVNIP2;1 determined by q-RT-PCR, in roots of seedlings of barley genotypes classified into four haplotypes on the basis of Southern and cDNA sequence analyses: Class 1, Clipper RFLP, ORF and uORF; 2, California Mariout RFLP, Clipper ORF and UORF; 3, Sahara RFLP and ORF/ Clipper UORF; 4, Sahara RFLP, ORF and UORF. Refer to Additional file 8: Table S5 for a list of barley genotypes included in each class. Transcript levels were normalised using the RNA housekeeping genes for tubulin, GAPDH and HSP70. c Relative translation efficiencies of a luciferase gene driven by an SP6 RNA polymerase promoter, when 5'UTR sequences from Clipper and Sahara HVNIP2; 1 are inserted immediately upstream of luciferase. Translation efficiency was measured as luciferase activity by luminescence, and is expressed relative to the activity of SP6 control luciferase DNA. Six transcription/translation reactions were set up for each construct, and four luciferase assays recorded for each reaction. Bar represents the $95 \%$ confidence interval for the difference between Clipper and Sahara 5'UTR sequences

The 2H QTL for B tolerance controls leaf symptom expression in the Clipper X Sahara 3771 population and is located close to the centromere [11]. Fine mapping in this region is difficult due to low recombination frequency. Furthermore, within the Clipper X Sahara 3771 DH population the QTL was poorly defined, largely because leaf symptoms could not be accurately assessed in lines carrying Sahara alleles at the other B tolerance loci. Additional background genetic effects might also contribute to an unclear phenotype in the $\mathrm{DH}$ lines. We identified seven $\mathrm{DH}$ lines that were recombinant within the $2 \mathrm{H}$ interval and backcrossed them to Clipper. $\mathrm{BC}_{1} \mathrm{~F}_{2}$ plants retaining the recombinant Sahara haplotype across the $2 \mathrm{H}$ region but with Clipper alleles at the $3 \mathrm{H}, 4 \mathrm{H}$ and $6 \mathrm{H}$ loci were selected by genotyping. Evaluation of leaf symptoms of $\mathrm{B}$ toxicity in $\mathrm{BC}_{1} \mathrm{~F}_{3}$ progeny clearly defined the interval for the $2 \mathrm{H}$ QTL to approximately $10.3 \mathrm{cM}$, between the markers GBMS0160 and Bmag381 (Additional file 6: Fig. S3, Panel A). We tested the markers contig888 and G9-138C in an attempt to characterise the barley genotypes at the $2 \mathrm{H}$ QTL region. G9-138C appeared to associate with a B tolerance phenotype. For this marker, the Sahara accessions and the line Ethiopia 756 shared a similar restriction pattern (Additional File 6: Fig. S3, Panel B).

\section{Prevalence and impact of B tolerance alleles in Australian barley varieties}

A set of 80 Australian barley varieties included in $\mathrm{Na}$ tional Variety Trial testing between 2008 and 2012 were screened for allele type at each of the four known B tolerance loci, leaf symptoms of B toxicity, and leaf B concentrations when grown in a glasshouse with an elevated supply of B. The data for each variety included in the screen are provided in Additional file 7: Table S4. A small number of varieties were heterogeneous for either the $H v N I P 2 ; 1$ or HvBot1 alleles, and were separated into sub-lines. The most common HvBot1 allele amongst the Australian barley varieties was the Clipper allele (67 genotypes). The Morex (10 genotypes) and Alexis (7 genotypes) alleles were also relatively common. None of the genotypes contained the Sahara
$H v N I P 2 ; 1$ allele on chromosome $6 \mathrm{H}$ or the Sahara variant of $\mathrm{HvBot} 2$ on chromosome $3 \mathrm{H}$.

Though few in number, genotypes in the study carrying single or multiple copies of the Sahara HvBot1 allele all had both low leaf B concentrations and few symptoms of B toxicity-induced leaf necrosis (Fig. 4; Additional file 7: Table S4). The remaining genotypes showed no clear relationship between allele type at HvBot1 and either leaf symptom score or leaf B concentrations. Similarly, we found no clear relationship of either B toxicity parameter to breeding origin (Additional file 7: Table S4). Overall there was only a weak positive relationship between leaf symptoms of $\mathrm{B}$ toxicity and leaf $\mathrm{B}$ concentration $\left(\mathrm{R}^{2}=\right.$ 0.235 ; Fig. 4), indicating that mechanism (s) other than exclusion of shoot B may be contributing to B tolerance in Australian barleys.

Two Australian cultivars, Navigator and Sloop Vic, have Sahara 3771 in their pedigrees and were found to contain the Sahara-derived HvBot1 gene duplication. Both cultivars have high levels of HvBot1 gene expression, and show tolerance to high soil B, with Sloop Vic bred specifically for this purpose. Sloop Vic was found to be genetically heterogeneous; within a single packet of seed we also identified seedlings carrying the Clipper HvBot1 allele. We designated sublines Sloop Vic_A and Sloop Vic_B, as carrying the Sahara HvBot1 duplication or the Clipper HvBot1 allele, respectively. Interestingly, while Sloop Vic_A had elevated levels of HvBot1 expression and reduced root $B$ concentrations relative to Sloop Vic_B and the parent cultivar Sloop, both Sloop Vic sublines showed low leaf symptoms of B toxicity (Figs. 4 and 5). Further genotyping revealed that both Sloop Vic_A and Sloop Vic_B have retained a segment from Sahara chromosome $2 \mathrm{H}$, carrying the gene or genes controlling leaf symptom expression.

In the varietal screen we included genotypes Parent 19 and Ethiopia 756, both containing a single copy of the Sahara HvBot1 allele. The two genotypes have been identified in Australian breeding programs to have good tolerance to high soil B. Parent 19 was provided to us by the University of Adelaide's Barley Breeding Program, while Ethiopia 756 was suggested for testing by breeders in Western Australia. In our screening Parent 19 and 


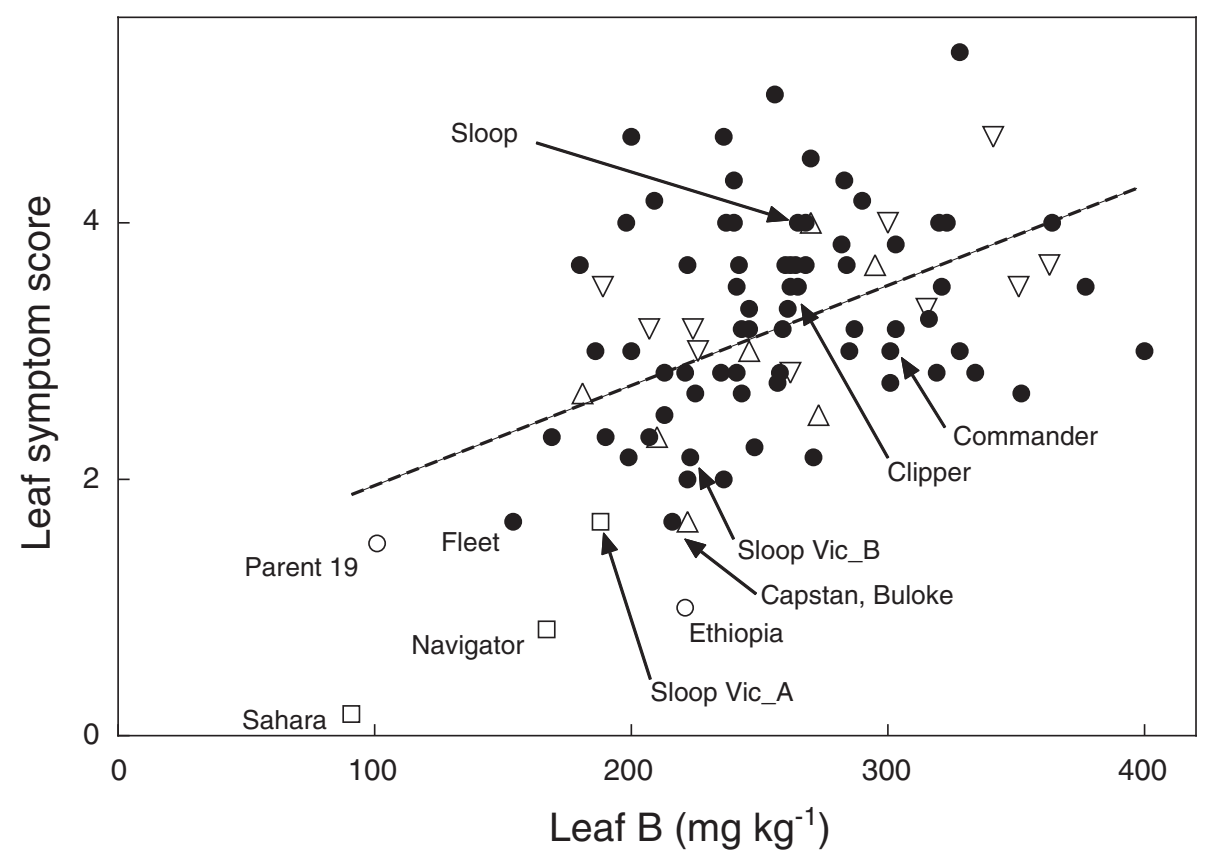

Fig. 4 Relationship between the severity of B toxicity-induced leaf necrosis and leaf B concentration for a set of current Australian barley varieties. Symptoms were assessed visually three times during the growth of plants to full maturity and an average score determined $(0=n o$ necrosis; $6=$ severe necrosis). The penultimate leaves from five tillers were sampled at mid-grain fill for each measurement of leaf B concentration. HvBot1 allele type for each variety is indicated (closed circles $=$ Clipper; open circles $=$ Sahara (single-copy); open squares $=$ Sahara (multi-copy); open upward triangles $=$ Alexis; open downwards triangles = Morex). The dashed line indicates a weak positive, linear correlation between the two parameters $\left(R^{2}=0.235\right)$

Ethiopia 756 also had both low leaf B concentrations and few leaf symptoms of B toxicity (Fig. 4). Although qRT-PCR and semi-q-RT-PCR experiments did not detect sizeable differences in HvBot1 expression between the Clipper and single-copy Sahara variants (Figs. 1b and 6a), we could not rule out a link between the presence of a single copy of the Sahara HvBot1 allele and a B tolerance phenotype. To determine the contribution of the Sahara HvBot1 allele to B tolerance in these genotypes, we generated $F_{2}$ populations from the crosses Parent $19 \times$ Clipper and Ethiopia $756 \times$ Clipper, and assessed leaf symptoms of B toxicity (Fig. 6b). Genetic segregation analysis did not reveal linkage between B tolerance determined by leaf symptom expression and the $H v B o t 1$ allele in either population (Fig. 6c). We developed a discriminating $\mathrm{KASP}^{\mathrm{m} \mathrm{m}}$ assay $(A B C 02403$; Additional file 5: Table S3) around sequence polymorphism between Clipper and the $\mathrm{B}$ tolerant parents of these populations, within the region on chromosome $2 \mathrm{H}$ associated with $\mathrm{B}$ tolerance in Sahara. Using this marker assay, we found an association between genotype at $2 \mathrm{H}$ and $\mathrm{B}$ tolerance in the Ethiopia $756 \times$ Clipper $\mathrm{F}_{2}$ segregants (Fig. 6c). $\mathrm{F}_{2}$ seedlings with a homozygous Ethiopia 756 genotype had, on average, $6.3 \%$ leaf necrosis on the first leaf. This was less than half the level of necrosis estimated on leaves of seedlings with a homozygous Clipper genotype, and a reduction of $33 \%$ compared to heterozygous seedlings (Fig. 6c). In the Parent $19 \times$ Clipper $\mathrm{F}_{2}$ population there was no association between the $2 \mathrm{H} \mathrm{KASP}^{\mathrm{m}}$ marker assay and a $\mathrm{B}$ tolerance phenotype.

In our varietal screen, several other barley cultivars also showed both low leaf symptoms of B toxicity and low leaf B concentrations, including Fleet, Capstan and Buloke (Fig. 4; Additional file 7: Table S4). We phenotyped a barley DH population (233 lines) derived from reciprocal crosses of Fleet with Commander. QTL analysis failed to reveal an association between leaf symptoms of B toxicity and any of the known $B$ tolerance loci (data not shown). Thus, the chromosomal regions responsible for B tolerance observed in Parent 19, Fleet and other cultivars with lower levels of tolerance remain unknown, and do not appear to correspond to any of the B tolerance QTL found in Sahara 3771.

\section{Discussion}

The identities of two genes conferring tolerance to high soil $B$ in barley have been determined $[12,13,16]$. One encodes HvNIP2;1, a transport protein belonging to the NIP family of aquaporins, and is found on chromosome $6 \mathrm{H}$. The other is on chromosome $4 \mathrm{H}$ and encodes a different kind of transporter, HvBot1. The tolerant alleles for both genes are present in a set of barley accessions from North Africa named Sahara, including the well-studied genotype Sahara 3771. A genetic study using a population derived 
(a)

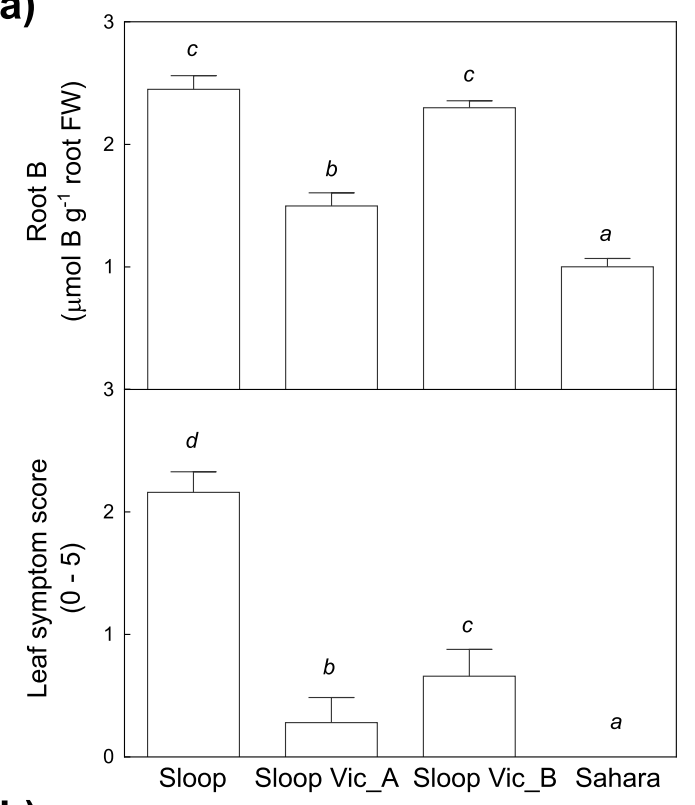

(b)

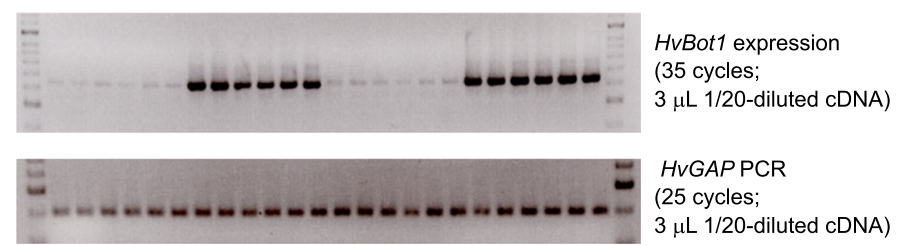

Fig. 5 Contribution of Sahara-derived B tolerance alleles on chromosomes $2 \mathrm{H}$ and $4 \mathrm{H}$ to B tolerance traits in the barley cultivar Sloop Vic. a Root B concentrations (upper panel) and leaf symptoms of B toxicity (lower panel) in the parental genotypes Sloop and Sahara, and two Sloop Vic sublines. Subline Sloop Vic_A contains the multi-copy Sahara HvBot1 allele on chromosome 4H, while Sloop Vic_B contains the Sloop (Clipper) HvBot1 allele. Both sublines carry the $2 \mathrm{H}$ B tolerance allele from Sahara. Seedlings were grown for 13 days in solution culture (11 days at 3 mM B). Within each panel, italicised letters above the bars denote significant differences between genotypes (Tukey's multiple comparisons test; $P=0.05$ ) b Semi-quantitative RT-PCR analysis of HvBot1 gene expression in roots of Sloop, Sloop Vic_A, Sloop Vic_B and Sahara seedlings grown for 11 days at $3 \mathrm{mM}$ B. Duplicate reactions were run for each of three cDNA samples for each genotype, and appear alongside each other in the image. The lower panel shows HVGAP fragment amplification, to indicate template concentration variability

from an intolerant barley variety, Clipper, and Sahara 3771, identified four chromosome locations associated with B tolerance, with all of the favourable alleles coming from Sahara 3771 [11]. In this study, we investigated the prevalence of these four alleles in barley germplasm, and the allelic diversity of $H v B o t 1$ and HvNIP2;1. Amongst the studied germplasm, we found that the Sahara lines contain a rare set of tolerance alleles, including a tandem duplication of $\operatorname{HvBot} 1(4 \mathrm{H})$, a critical 5'UTR SNP in the coding sequences of $H v N I P 2 ; 1(6 \mathrm{H})$, a deletion in the gene $H v B o t 2$ that is located under the $3 \mathrm{H}$ tolerance locus, and a rare haplotype across the 2H QTL region.

Tolerance due to HvBot1 (chromosome 4H) was originally attributed to either greater functionality of the transporter as a result of sequence differences between the Sahara and Clipper HvBot1 coding sequences, or higher levels of expression [12]. In addition to promoter sequence differences, tandem duplication of
HvBot1 in the genome of Sahara has created an estimated four copies. Our study indicates that this duplication occurred relatively recently in barley evolution, and is rare. Southern analysis showed that the duplication is only present in Sahara 3771, eight other genotypes also accessioned with the Sahara name, and two Australian cultivars that contain Sahara 3771 in their pedigree. Sequence analysis of many genotypes in this study also revealed that the original published $H v B o t 1$ sequence for Clipper contained a sequencing error. There are in fact no residue differences between the alleles from Clipper and Sahara 3771, and both alleles confer a similar level of B tolerance to yeast when heterologously expressed (Fig. 1). All HvBot1 sequences lodged with GenBank have been amended and updated accordingly. Thus, differences between the two alleles are entirely attributable to expression level differences.

We identified seven $H v B o t 1$ CDS alleles in total. Only one of these, found in two Japanese cultivars Haruna 
(a)

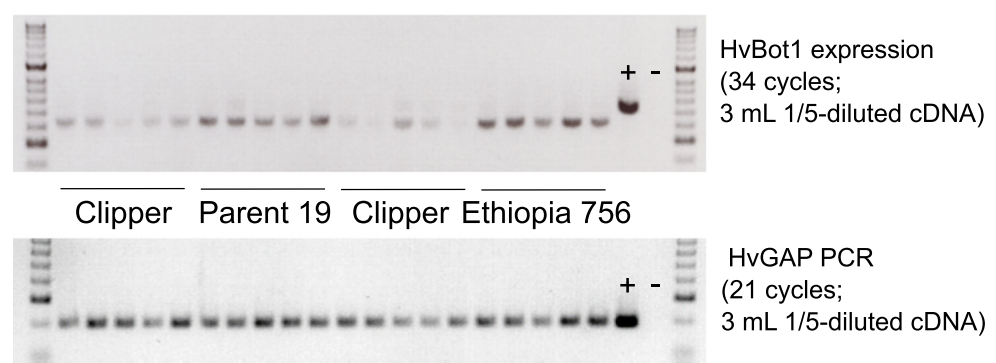

(b)

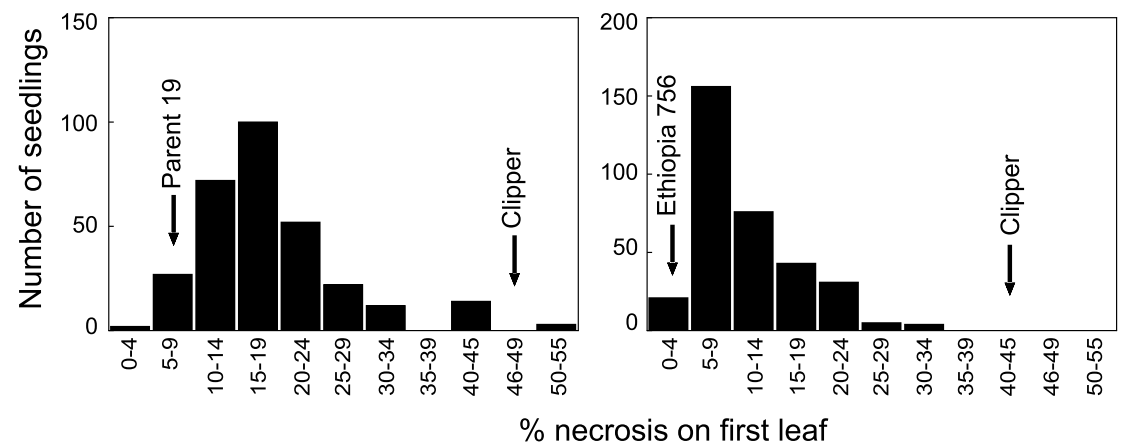

(c)

$\%$ necrosis on first leaf
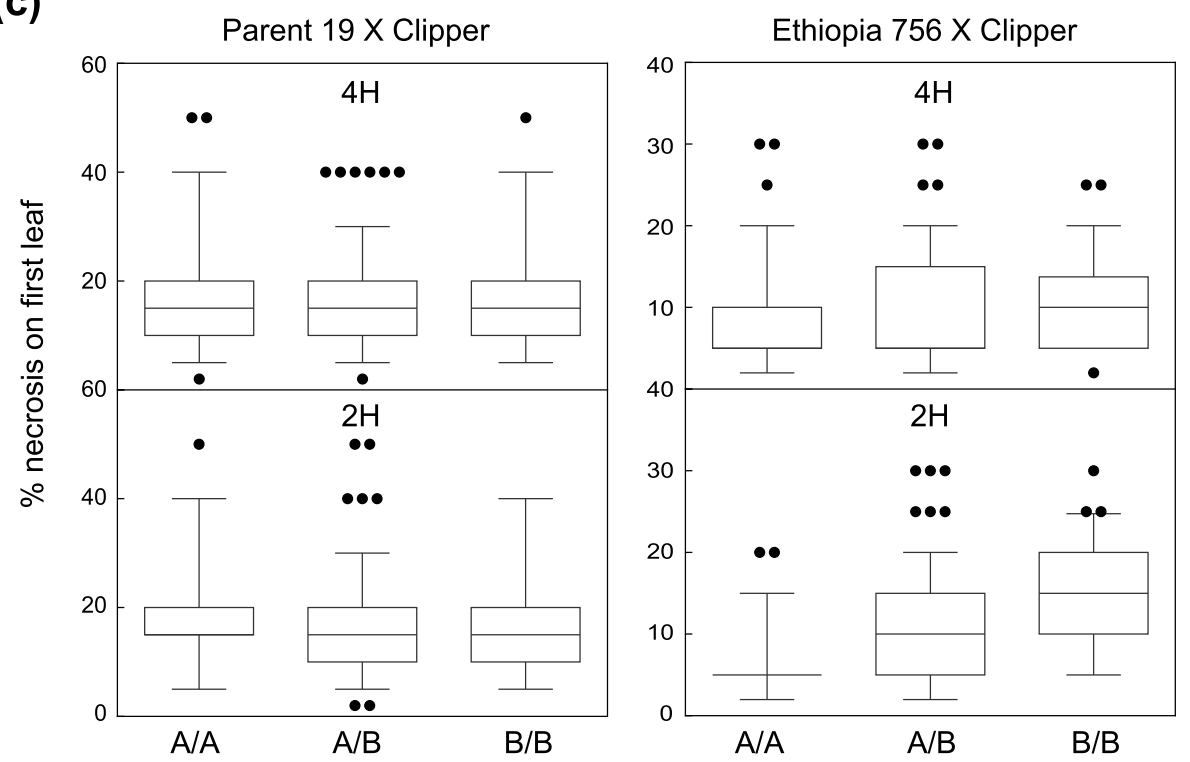

Fig. 6 A single copy of the Sahara HvBot1 allele on chromosome $4 \mathrm{H}$ does not contribute to B tolerance in barley. a Semi-quantitative RT-PCR analysis of HvBot1 expression in roots of Clipper, Parent 19 and Ethiopia 756 seedlings. Each sample is derived from a single seedling grown in nutrient solution containing an additional $3 \mathrm{mM} \mathrm{B}$ for 15 days. The lower panel shows HVGAP fragment amplification, to indicate template concentration variability. $+=$ positive control using HvGAP PCR product as template; - = water control. b Distribution of percentage leaf necrosis in $\mathrm{F}_{2}$ progeny derived from the crosses Parent $19 \times$ Clipper (left panel; $N=294$ ) and Ethiopia $756 \times$ Clipper (right panel; $N=337$ ). Seedlings were grown for 15 days in nutrient solution containing $3 \mathrm{mM}$ B. Average necrosis for each parent is also indicated. $\mathbf{c}$ Leaf necrosis of $\mathrm{F}_{2}$ plants in the two populations segregating for HvBot1 allele (chromosome 4H; upper panels) and a KASP ${ }^{\mathrm{TM}}$ marker linked to the $2 \mathrm{H}$ B tolerance locus (lower panels). In all panels, the Parent 19 or Ethiopia allele is represented by A, and the Clipper allele represented by B. The boundaries of the boxes indicate $75^{\text {th }}$ and $25^{\text {th }}$ centiles, lines within mark the median, bars above and below the boxes indicate $90^{\text {th }}$ and $10^{\text {th }}$ centiles, and outliers are shown as circles. The percentage variation $\left(R^{2}\right)$ explained in each population by marker genotype is also shown

Nijo and Amagi Nijo, showed reduced function when expressed in yeast. This is due to a residue substitution at position 234 in the non-functional Haruna Nijo allele, of histidine for leucine (Fig. 1c). All single-copy HvBot1 alleles were expressed at similarly low levels, including the single-copy Sahara HvBot1 allele (Fig. 1b). Genomic sequencing of a number of single-copy Sahara HvBot1 genotypes failed to identify any sequence differences 
between the single-copy and multi-copy Sahara alleles, or between copies present in Sahara. Moreover, a line derived from the Clipper X Sahara $3771 \mathrm{DH}$ population and with a recombination within the HvBot1 gene cluster, showed a dependency of $H v B o t 1$ gene expression level on the number of Sahara HvBot1 copies present (Fig. 2). We conclude that the increased expression of HvBot 1 found in Sahara is due to the duplication of this gene. A number of other specific examples of gene copy number expansion occurring through evolution have been described in plant genomes, which have also resulted in increased gene expression [17-19]. Recent whole genome analyses have revealed that copy number variation is common in plants, and appears to be biased towards genes involved in abiotic and biotic stress responses [20, 21].

Further, we propose that duplication of the Sahara $H v B o t 1$ allele is responsible for B tolerance at the $4 \mathrm{H}$ locus. Segregation analysis in two independent $\mathrm{F}_{2}$ barley populations (Parent $19 \times$ Clipper; Ethiopia $756 \times$ Clipper) failed to demonstrate linkage between the presence of a single copy of the Sahara HvBot1 allele and $\mathrm{B}$ tolerance as determined by leaf symptom expression (Fig. 6). By contrast, in the original genetic study the duplicated HvBot1 allele from Sahara was associated with leaf symptom development, as well as a number of other traits associated with B tolerance [11].

We also examined diversity in coding sequence and gene expression level for $H v N I P 2 ; 1$, the gene underlying a major QTL in Sahara 3771 on chromosome 6H [13], revealed that the $H v N I P 2 ; 1$ sequences were highly conserved amongst the studied germplasm. We identified only two SNPs in coding sequence of this gene; one synonymous SNP that was present in around half of the genotypes examined and could not be related to a B tolerance phenotype, and a second SNP in the 5'UTR that was unique to the set of nine Sahara accessions (Fig. 3a). We propose that the 5'UTR SNP is causative of B tolerance in Sahara. An in vitro transcription/translation assay showed reduced luciferase activity in the presence of the 5'UTR derived from Sahara HvNIP2;1 (Fig. 3c), suggesting that a small uORF created by the Sahara 5'UTR SNP interferes with translation of $H v N I P 2 ; 1$. In our previous study we detected a modest reduction in $H v N I P 2 ; 1$ transcript levels in root tissues of B-treated Sahara 3771 compared to Clipper [13]. However, in broadening the analysis of expression to a wider range of genotypes, we were not able to reproduce these differences (Fig. 3b). Future experiments to quantify active protein levels in roots of Sahara and other, intolerant barley genotypes will help to confirm if the uORF in Sahara inhibits HvNIP2;1 translation. Upstream ORFs are common elements of plant transcript sequences and have been shown to mediate translation for a number of genes (reviewed in [22]), including transcription factors $[23,24]$ and enzymes involved in polyamine biosynthesis [25].

It has been suggested that when using Sahara as a breeding parent, there may be linkage drag of unfavourable traits together with the $4 \mathrm{H}$ allele, or pleiotropic effects of the $4 \mathrm{H}$ tolerance allele in different backgrounds $[26,27]$. However, we identified two released barley varieties carrying the $4 \mathrm{H}$ allele from Sahara, Navigator and Sloop Vic (Additional file 7: Table S4), demonstrating there are unlikely to be severe penalties in either yield or quality traits associated with the introgression of the HvBot1 gene duplication. We investigated by genotype analysis the extent of the Sahara-derived $4 \mathrm{H}$ segment in Navigator (WI4262; released 2009), and estimate it to cover a region of at least $14 \mathrm{cM}$. While developed for cultivation in higher rainfall, long-season environments not typically associated with B toxicity, Navigator consistently shows low levels of leaf symptom expression when challenged with high B (this study and [28]).

The cultivar Sloop Vic was specifically selected for B tolerance [29]. We identified genetic heterogeneity for the $4 \mathrm{H}$ locus in this variety (Fig. 5), but also the presence of a chromosomal segment from Sahara that includes the $2 \mathrm{H} \mathrm{B}$ tolerance allele. The two other tolerance alleles from Sahara $(3 \mathrm{H}$ and $6 \mathrm{H})$ were not retained in Sloop Vic. Our findings suggest that the $2 \mathrm{H}$ allele is highly useful for imparting $\mathrm{B}$ tolerance. We also identified another unadapted six-row barley, Ethiopia 756, as an alternative source of the $2 \mathrm{H}$ tolerance allele. Ethiopia 756 shares the same haplotype for a number of markers across the $2 \mathrm{H}$ interval with Sahara, including a rare G9-138C allele. Segregation analysis in a population derived from Ethiopia 756 crossed with Clipper revealed an association between the critical $2 \mathrm{H}$ region in Ethiopia 756 and leaf symptoms of B toxicity (Fig. 6). In an alternative population derived from Parent 19 and Clipper the same region was not linked with B tolerance.

\section{Conclusions}

This study of allelic diversity for the four known boron tolerance loci revealed that Sahara 3771 has a unique set of boron tolerance genes, rare amongst germplasm. It allowed us to identify the causative features of two of the genes responsible for B tolerance in Sahara. These are a tandem duplication of HvBot1 and a short, upstream open reading frame in the coding sequences of $H v N I P 2 ; 1$. Our findings facilitate the development of markers for tracking B tolerance in barley with greater specificity, and we designed three KASP ${ }^{\mathrm{m}}$ marker assays for tracking tolerance derived from Sahara. Our detection of the $2 \mathrm{H}$ locus in Ethiopia 756 is the first reported 
validation of the Sahara 3771 B tolerance QTL in another population.

The genetic locations controlling B tolerance in Parent19, as well as lower levels of tolerance in cultivars such as Fleet, Buloke and Capstan that were observed in our study and in the field by others [6,7], remain unknown; selection for B tolerance derived from these sources can only be made on the basis of phenotype. However, the genotypes Ethiopia 756 and Sloop Vic may be used in breeding programs as sources of B tolerance attributable to a significant QTL for leaf symptom expression on chromosome $2 \mathrm{H}$, and may be valuable alternatives to Sahara.

\section{Methods}

Plant material

Seed for most genotypes of barley (Hordeum vulgare L.) was obtained from either the Australian Grains Genebank or from our own collections. Seed of Parent 19 and a number of ICARDA genotypes showing $B$ tolerance in the field was provided by Drs Jason Eglinton and Stewart Coventry, The University of Adelaide's Barley Breeding Program. The varieties Compass, Flinders, Grange, Henley, LaTrobe, SY Rattler and Westminster were obtained from Dr Hugh Wallwork (South Australian Research and Development Institute), and Macumba was obtained from Amanda Box (The University of Adelaide).

\section{Allele diversity analyses}

Seeds were germinated on moist filter paper in the laboratory. After 4-7 days, whole roots and shoots from five uniform seedlings for each genotype were pooled and snap-frozen in liquid nitrogen, for RNA and DNA extractions, respectively. Pooling from a number of individual seedlings facilitated the detection of genetic heterogeneity within lines. The 65 genotypes included for allele diversity analysis are listed in Additional file 1: Table S1.

RNA was extracted using TRIzol (Invitrogen). For cDNA synthesis, we used Superscript III Reverse Transcriptase (Invitrogen); samples for quantitative real-time reverse transcription polymerase chain reaction (q-RT-PCR) were DNase-treated prior to cDNA synthesis using DNA-Free (Ambion, USA). Coding sequence for HvBot1 and $H v N I P 2 ; 1$ was amplified from cDNA obtained from 68 genotypes, and Sanger sequenced at the Australian Genome Research Facility (AGRF; Adelaide). q-RT-PCR analysis of expression of $H v B o t 1$ and $H v N I P 2 ; 1$ was performed as previously described [30], using gene-specific primers (Additional file 5: Table S3). For some experiments, semi-quantitative reverse transcription PCR (semiq-RT-PCR) was performed on cDNAs, where we used
HvGAP amplification (Additional file 5: Table S3) to indicate between-sample variation in template concentration.

Genomic DNA was isolated using phenol-chloroform extraction, digested with the restriction enzyme Dra I and the products separated by gel electrophoresis before transfer to a nylon membrane for Southern hybridisation using standard methods. Membranes were probed with radio-labelled nucleic acid fragments of the $\mathrm{B}$ tolerance genes HvBot1 and HvNIP2;1, and the putative tolerance gene HvBot2 (co-locating with a tolerance QTL on chromosome $3 \mathrm{H})$. Several probes located within the region of the $2 \mathrm{H}$ B tolerance QTL were also utilised. Probe details are included in Additional file 5: Table S3. Membranes were stripped with a $0.1 \%$ sodium dodecyl sulphate (SDS), $2 \mathrm{mM}$ ethylenediaminetetraacetic acid (EDTA) solution heated to boiling point between each sequential hybridisation.

\section{Heterologous expression and in vitro transcription/ translation experiments}

Open reading frames for each of the identified HvBot1 alleles were PCR-amplified from cDNA prepared from roots of the barley genotypes Sahara 3771, Clipper, Haruna Nijo, WI4304, Alexis and Tadmor using primers listed in Additional file 5: Table S3, and products were cloned in the Gateway entry vector $p C R 8$ (Invitrogen, Carlsbad, CA, USA). Sequences of the inserts were verified by Sanger sequencing before recombining into the destination vector pYES3.DEST (Invitrogen) for yeast expression. The Sahara $H v B o t 1$ expression vector was also used as a template for in vitro site-directed mutagenesis (Quikchange II SiteDirected Mutagenesis kit, Stratagene, La Jolla CA, USA) using specific primer pairs detailed in Additional file 5: Table S3. Two variants of HvBot1 were created, each with a single residue substitution of either Leu234His or Thr541Met. Both substitutions are present in the nonfunctional Haruna Nijo HvBot1 allele. Experiments for functional assessment of the HvBot1 alleles and variants in yeast (Saccharomyces cerevisiae) were performed as previously described [31].

5'UTR sequences from HvNIP2;1 genes of Clipper and Sahara were amplified from cDNA using primers listed in Additional file 5: Table S3. The PCR products and SP6 control luciferase vector DNA (Promega, USA) were digested with restriction enzymes Not I and Bam HI. Digested products were then cloned into the linearised SP6 vector, between the SP6 RNA polymerase promoter and the firefly (Photinus pyralis) luciferase gene. Insert sequences were confirmed by Sanger sequencing. We then used a TNT Coupled Wheat Germ Extract System (Promega, USA) to perform in vitro transcription/translation reactions using SP6 control DNA and the modified vector. We made six reactions for each construct. Translation of luciferase protein for each reaction was 
measured as luciferase activity, by recording luminescence using a POLARstar Optima plate reader (BMG LabTech) programmed to perform multiple reads on each sample at $1 \mathrm{~s}$ intervals for a total of approximately $2 \mathrm{~min}$. All plotted data were linear with slopes close to zero, and were extrapolated to time 0 to determine initial rates of activity. Four luminescence assays were recorded for each transcription/translation reaction.

\section{Screening of current varieties}

Seeds were sown into 50:50 UC mix: coco peat potting medium, in $25 \mathrm{~cm}$ diameter pots without replication. Following germination, each pot was thinned to three uniform seedlings. Plants were grown through to maturity during spring, in a temperature-controlled greenhouse (day/night average temperatures of $23{ }^{\circ} \mathrm{C} / 19{ }^{\circ} \mathrm{C}$ ) with natural lighting. Pots were moved during the growing period to avoid positional effects on light interception or transpiration, which might influence the development of symptoms of B toxicity. Plants were watered regularly with tap water, and fertilised twice with a multi-element, slow-release fertiliser. Tap water delivered to the University of Adelaide's Waite Campus between August and December 2013 contained B concentrations of $0.1-0.5 \mathrm{mg} \mathrm{B} \mathrm{L}{ }^{-1}$, which was sufficient to induce $\mathrm{B}$ toxicity-attributable leaf necrosis in intolerant barley varieties in this experiment. We have consistently observed B toxicity symptoms in barley plants grown across the Waite Campus since 2012, co-incident with elevated $B$ concentrations in tap water (data not supplied). In this experiment, symptoms of B toxicity were assessed visually three times during the growth of plants to full maturity and an average score determined $(0=$ no necrosis; 6 $=$ severe necrosis). The penultimate leaves from five tillers were sampled at mid-grain fill, oven-dried and chopped finely with scissors. A sub-sample of dried leaf tissue was acid-digested for analysis of B concentration by Radial View Inductively Coupled Plasma-Optical Emission Spectrometry (ICP-OES) [32]. Selected varieties were grown hydroponically with high $B$ using a nutrient solution described in [12]. B concentrations in root tissues of these plants were determined using an azomethine- $\mathrm{H}$ colorimetric assay [33].

\section{Development of KASP ${ }^{\mathrm{TM}}$ markers to detect the Sahara} 3771 allele at the HvBot1, HvBot2 and HvNIP2;1 loci

KASP $^{\mathrm{m}}$ markers were designed based on polymorphisms between Sahara 3771 and B intolerant cultivars at the B tolerance loci HvBot1, HvBot2 and HvNIP2;1 (Additional file 4: Table S2). The markers wri57 and wri59 (for $H v B o t 1$ and $H v N I P 2 ; 1$, respectively) assay SNP polymorphisms between the Sahara accessions and B intolerant lines, while the wri58 marker (for HvBot2) assays a deletion present only in Sahara accessions. All primer sets were designed using Kraken (LGC Limited, London, UK) with default parameters. Assays were validated in the parental lines and a set of varieties for which alleles were previously determined using an automated SNPLine system (LGC Limited, London, UK).

\section{$F_{2}$ segregant analysis of $B$ toxicity symptoms}

Crosses were made using two barley lines with low levels of leaf B toxicity symptoms as female parents (Parent 19 and Ethiopia 756), and the intolerant variety Clipper as the male parent. $F_{1}$ hybrids from these crosses were confirmed by genotyping using the $x$ Bot 1 CAPS marker (Additional file 5: Table S3). $\mathrm{F}_{2}$ progeny were germinated on moist filter paper, and transferred to aerated nutrient solution containing $3 \mathrm{mM} \mathrm{B}$. Seedlings were grown for $15 \mathrm{~d}$ in a growth chamber $\left(18{ }^{\circ} \mathrm{C} / 15{ }^{\circ} \mathrm{C}\right.$ and $50 \% / 70 \%$ humidity day/night settings, with a $12 \mathrm{~h}$ photoperiod and light intensity of approx. $200 \mathrm{~mol} \mathrm{~m}^{-2} \mathrm{~s}^{-1}$ at plant height). Individual seedlings were then scored for percentage necrosis on the first leaf, and sampled for DNA isolation using a freeze-dried method of extraction [34]. Material was genotyped at the $4 \mathrm{H}$ and $2 \mathrm{H} \mathrm{B}$ tolerance loci using the $\mathrm{KASP}^{\mathrm{m}}$ assays wri57 (Additional file 4: Table S2) and ABC02403 (Additional file 5: Table S3), respectively. The $2 \mathrm{H} \mathrm{KASP}^{\mathrm{m}}$ marker was designed and validated as discriminating between Clipper and the $\mathrm{B}$ tolerant parents, and verified by genotyping of selected recombinant lines to reside within the defined QTL interval. Association between genotype and phenotype for each population and each of the $4 \mathrm{H}$ and $2 \mathrm{H}$ regions was analysed by separate one-way ANOVA.

\section{Availability of supporting data}

The data sets supporting the results of this article are included within the article and its additional files. $\mathrm{Nu}$ cleic acid sequences described in this research have been lodged with GenBank: [GenBank:EF660435, GenBank: EF660437, GenBank:KR605456, GenBank:KR605457, Gen Bank:KR605458, GenBank:KR605459, GenBank:KR605460, GenBank:KR605461, GenBank:KR817581].

\section{Additional files}

Additional file 1: Table S1. Barley genotypes used for initial characterisation of allele diversity at each of four boron tolerance loci (PDF $233 \mathrm{~kb}$ )

Additional file 2: Figure S1. Nine barleys accessioned with the name Sahara contain the rare HvBot1 gene duplication. (a) Southern analysis of allele type and gene duplication at HvBot1, for a representative set of barley genotypes. Lane 1, Clipper; 2, Sahara 3771; 3; Alexis; 4, Amagi Nijo; 5, Arapiles; 6, Atlas; 7, Barque; 8, California Mariout; 9, Chebec; 10, CM72; 11, Commander; 12, Flagship; 13, Fleet; 14, Franklin; 15, Gairdner; 16, Galleon; 17, Golden Promise; 18, Halcyon; 19, Haruna Nijo; 20, Clipper; 21, Sahara 3771; 22, Keel; 23, Morex; 24, Mundah; 25, Sahara 3763; 26, Sahara 3764; 27, Sahara 3765; 28, Sahara 3766; 29, Sahara 3767; 30, Sahara 3768; 31, Sahara 3769; 32, Sahara 3770, 33, Schooner; 34, Skiff; 35, Sloop; 36, 
Steptoe; 37, Tadmor; 38, Tokak. Genomic DNA was digested with Dra l, and probed with a $\left.{ }^{32} \mathrm{P}\right]$-labelled nucleic acid fragment of HvBot1 derived from Clipper (Additional File 5: Table S3). (b) Leaf symptom scores (upper panel) and shoot B concentrations (lower panel) of nine Sahara barley accessions grown in nutrient solutions with $5 \mathrm{mM}$ additional B (PDF $1165 \mathrm{~kb}$ )

Additional file 3: Figure S2. The Haruna Nijo variant of HvBot1 is non-functional. (a) Growth of yeast expressing HvBot1 alleles relative to either an empty vector control (top two plates only) or clones expressing the Sahara HvBot1 allele, on low B media (left) or at high $B$ (right). Three independent clones for each allele were cultured on each plate; clones are positioned vertically and spots across the plates represent $10 \mathrm{~L}$ of $10^{-0}, 10^{-1}, 10^{-2}$ and $10^{-3}$ dilutions from a starting culture containing approximately $3 \times 10^{7}$ cells $\mathrm{L}^{-1}$. Photographs were taken after $2 \mathrm{~d}$ (low B) or $4 \mathrm{~d}\left(20 \mathrm{mM}\right.$ B) incubation at $30^{\circ} \mathrm{C}$. With the exception of the Haruna Nijo allele, all variants conferred a similar level of tolerance to high B in yeast to the Sahara HvBot1 allele. (b) Concentration of $B$ in roots of barley seedlings (cvs. Clipper, Haruna Nijo, Amagi Nijo and Sahara) grown for 11 days in hydroponics supplemented with $3 \mathrm{mM}$ $B(N=5, \pm s d)$. The genotypes Haruna Nijo and Amagi Nijo carry the non-functional Haruna Nijo HvBot1 allele at the $4 \mathrm{H}$ B tolerance QTL (PDF $369 \mathrm{~kb}$ )

Additional file 4: Table S2. KASPTM marker assays designed around Sahara-specific gene coding sequence features for HvBot1, HvNIP2;1 and HvBot2. These markers are suitable for tracking the introgression of the $4 \mathrm{H}$, $6 \mathrm{H}$ and $3 \mathrm{H} \mathrm{B}$ tolerance alleles, respectively, from the Sahara accessions (PDF $386 \mathrm{~kb}$ )

Additional file 5: Table S3. Primers, PCR markers and probes used in this study (PDF $350 \mathrm{~kb}$ )

Additional file 6: Figure S3. Resolution of the $2 \mathrm{H}$ interval containing a QTL for leaf symptom expression in a Clipper X Sahara 3771 barley doubled haploid (DH) population. (a) The graph shows leaf symptom scores $\left(0=\right.$ no symptoms; $5=$ severe leaf necrosis) for $2-4 \mathrm{BC}_{1} \mathrm{~F}_{2}$ families of each of seven lines derived from selected $\mathrm{DH}$ lines backcrossed to Clipper and selected for intolerant alleles at each of the $3 \mathrm{H}, 4 \mathrm{H}$ and $6 \mathrm{H} \mathrm{B}$ tolerance QTL. Each of the lines was also selected for homozygosity of the $2 \mathrm{H}$ recombinant haplotype. Symptom scores for the parental genotypes (Clipper and Sahara 3771), and eight additional DH lines with unequivocal $2 \mathrm{H}$ genotypes and Clipper alleles at the other loci are shown for comparison (open symbols). The schematic below shows genotypes for each of the lines across the $2 \mathrm{H}$ interval, with open and shaded bars representing Clipper- and Sahara-derived chromosome segments, respectively. Markers within the interval are in bold type, and span a region of $10.3 \pm 2.9 \mathrm{cM}$. The KASP ${ }^{\mathrm{TM}}$ marker designed to discriminate between Clipper and Parent 19 or Ethiopia 756 and used for genotyping $F_{2}$ segregants from Parent $19 \times$ Clipper and Ethiopia $756 \times$ Clipper populations in Fig. 6 is shown in red type. (b) Southern analysis of G9-138C allele type for a representative set of barley genotypes, including the Sahara accessions (lanes 6-13) and Ethiopia 756 (lane 23). Lanes 1 and 20; Clipper. Lanes 2 and 21; Sahara 3771 (PDF $640 \mathrm{~kb}$ )

Additional file 7: Table S4. Breeding origin, genotype at HvBot1, and B tolerance phenotype of 80 current or recent Australian barley varieties grown to maturity in a glasshouse with an elevated supply of B. Breeding lines Parent 19 and Ethiopia 756 were also included in the screen, as well as Sahara 3771 and Clipper control genotypes. All varieties had a Clipper (B-intolerant) allele at chromosomes $6 \mathrm{H}$ and $3 \mathrm{H}$, while at $2 \mathrm{H}$ only Sloop Vic_A, Sloop Vic_B and Ethiopia 756 possessed the tolerant (Sahara) allele. Varieties are listed in order of increasing severity of leaf symptoms expression. Symptoms were assessed visually three times during the growth of plants to full maturity and an average score determined $(0=$ no necrosis; $6=$ severe necrosis). The penultimate leaves from five tillers were sampled at mid-grain fill for determination of leaf B concentration (PDF $464 \mathrm{~kb}$ )

Additional file 8: Table S5. Barley genotypes included in each (a) HvBot1 allele class and (b) HvNIP2;1 haplotype class, for gene expression analyses data presented in Figs. $1 \mathrm{~b}$ and 3b, respectively (PDF $301 \mathrm{~kb}$ )

\section{Competing interests}

The authors declare that they have no competing interests.

\section{Authors' contributions}

$\mathrm{JH}$ and MP jointly conceived of, designed and co-ordinated the study, and analysed the data. JH performed the phenotyping, genotyping, gene sequence and gene expression analysis experiments, heterologous expression of the HvBot1 variants in yeast and in vitro transcription/translation of HvNIP2;1, and drafted the manuscript. MP assisted with phenotyping and genotyping experiments, carried out the genomic Southern analyses and sequence analyses, and helped draft the manuscript. MG designed and validated the Sahara-specific KASP ${ }^{\text {TM }}$ marker assays and helped draft the manuscript. MO identified and characterised HvBot2. JR performed in vitro mutagenesis and heterologous expression of the Haruna Nijo HvBot1 allele. TS participated in the design of the study, interpretation of the results and drafting of the manuscript. All authors read and approved the final manuscript.

\section{Acknowledgements}

We thank Alison Hay for technical assistance, Ute Baumann, Carolyn Schultz and Nadim Shadiac for their advice regarding the HvNIP2;1 in vitro transcription/translation experiment, and Penny Tricker for critically reading the manuscript. Tefera Angessa (University of Western Australia) suggested inclusion of genotype Ethiopia 756 in screening for B tolerance, and Stewart Coventry (The University of Adelaide's Barley Breeding Program) provided seed of Parent 19 and a selection of ICARDA lines observed to have B tolerance in field screens. Waite Analytical Services performed the acid extraction and ICP-OES analysis of B concentrations in leaf material, and Yuan $\mathrm{Li}$ and Stephen Fletcher performed the q-RT-PCR experiments. We also acknowledge Tim March for running a QTL analysis on genotyping-by-sequencing marker data for a barley DH population derived from reciprocal crosses of Fleet and Commander. This research was jointly funded by the Australian Research Council, the Grains Research and Development Corporation, The University of Adelaide and the South Australian Government.

\section{Author details}

${ }^{1}$ Australian Centre for Plant Functional Genomics and The University of Adelaide, Adelaide, South Australia, Australia. ${ }^{2}$ Present address: University of Florida Agronomy Department, Gainesville, Florida, USA. ${ }^{3}$ Present address: South Australian Research and Development Institute, Adelaide, South Australia, Australia.

Received: 18 June 2015 Accepted: 6 September 2015 Published online: 26 September 2015

\section{References}

1. Cartwright B, Zarcinas BA, Mayfield AH. Toxic concentrations of boron in a red-brown earth at Gladstone. South Australia Aust J Soil Res. 1984;22:261-72. doi:10.1071/SR9840261.

2. McDonald GK, Eglinton JK, Barr AR. Assessment of the agronomic value of QTL on chromosomes $2 \mathrm{H}$ and $4 \mathrm{H}$ linked to tolerance to boron toxicity in barley (Hordeum vulgare L.). Plant Soil. 2010;326:275-90. doi:10.1007/ s11104-009-0006-1.

3. Reid R, Fitzpatrick K. Influence of leaf tolerance mechanisms and rain on boron toxicity in barley and wheat. Plant Physiol. 2009;151:413-420. doi: http://dx. doi. org/ 10.1104/ pp.109.141069

4. Nuttall JG, Hobson KB, Materne M, Moody DB, Munns R, Armstrong RD. Use of genetic tolerance in grain crops to overcome subsoil constraints in alkaline cropping soils. Soil Res. 2010;48:188-199. doi:10.1071/SR09081

5. Mertens J, Van Laer L, Salaets P, Smolders E. Phytotoxic doses of boron in contrasting soils depend on soil water content. Plant Soil. 2011;342:73-82. doi:10.1007/s11104-010-0666-X.

6. Paynter B, Hills A, Gupta S, Collins S, Dhammu H, Malik R, Trainor G. 2015 Barley Variety Sowing Guide for Western Australia. Bulletin 4860, WA Department of Agriculture and Food; 2014. ISSN: 18337366.

7. Wheeler R. Barley variety sowing guide 2015. In: South Australian Sowing Guide 2015. South Australian Research and Development Institute; 2014. pp. $16-20$

8. Nable RO. Resistance to boron toxicity amongst several barley and wheat cultivars: A preliminary examination of the resistance mechanism. Plant Soil. 1988;112:45-52. doi:10.1007/BF02181751.

9. Paull JG, Cartwright B, Rathjen AJ. Responses of wheat and barley genotypes to toxic concentrations of soil boron. Euphytica. 1988;39:137-44. doi:10.1007/BF00039866. 
10. Waterhouse WL. Studies in the inheritance of resistance to leaf rust, Puccinia anomala Rostr., in crosses of barley. I. Reprinted from the J Proc Royal Soc New South Wales, 1927; Vol. LXI:218-247.

11. Jefferies S, Barr AR, Karakousis A, Kretshmer JM, Manning S, Chalmers KJ, et al. Mapping of chromosome regions conferring boron toxicity tolerance in barley (Hordeum vulgare L.). Theor Appl Genet. 1999;98:1293-303. doi:10.1007/s001220051195.

12. Sutton T, Baumann U, Hayes J, Collins NC, Shi B-J, Schnurbusch T, et al. Boron toxicity tolerance in barley arising from efflux transporter amplification. Science. 2007;318:1446-9. doi:10.1126/science.1146853.

13. Schnurbusch T, Hayes JE, Hrmova M, Baumann U, Ramesh SA, Tyerman SD, et al. Boron toxicity tolerance in barley through reduced expression of the multifunctional aquaporin HvNIP2;1. Plant Physiol. 2010;153:1706-15. doi:10.1104/pp.110.158832.

14. Pallotta M, Schnurbusch T, Hayes J, Hay A, Baumann U, Paull J, et al. Molecular basis of adaptation to high soil boron in wheat landraces and elite cultivars. Nature. 2014;514:88-91. doi:10.1038/nature13538.

15. Öz MT. Microarray based expression profiling of barley under boron stress and cloning of $3 \mathrm{H}$ boron tolerance gene. PhD Thesis, Middle East Technical University. 2012. Available: https://etd.lib.metu.edu.tr/upload/12614203/ index.pdf

16. Reid R. Identification of boron transporter genes likely to be responsible for tolerance to boron toxicity in wheat and barley. Plant Cell Physiol. 2007:48:1673-8. doi:10.1093/pcp/pcm159.

17. Hanikenne M, Talke IN, Haydon MJ, Lanz C, Nolte A, Motte P, et al. Evolution of metal hyperaccumulation required cis-regulatory changes and triplication of HMA4. Nature. 2008:453:391-5. doi:10.1038/nature06877.

18. Cook DE, Lee TG, Guo X, Melito S, Wang K, Bayless AM, et al. Copy number variation of multiple genes at Rhg1 mediates nematode resistance in soybean. Science. 2012;338:1206-9. doi:10.1126/science.1228746.

19. Maron LG, Guimarães CT, Kirst M, Albert PS, Birchler JA, Bradbury PJ, et al. Aluminum tolerance in maize is associated with higher MATE1 gene copy number. Proc Nat Acad Sci. 2013;110:5241-6. doi:10.1073/pnas.1220766110.

20. McHale LK, Haun WJ, Xu WW, Bhaskar PB, Anderson JE, Hyten DL et al. Structural variants in the soybean genome localize to clusters of biotic stress-response genes. Plant Physiol. 2012;159:1295-1308. doi:10.1104/ pp.112.194605

21. Muñoz-Amatriaín M, Eichten SR, Wicker T, Richmond TA, Mascher M Steuernagel B, et al. Distribution, functional impact, and origin mechanisms of copy number variation in the barley genome. Genome Biol. 2013;14:R58. doi:10.1186/gb-2013-14-6-r58.

22. Von Arnim AG, Jia Q, Vaughn JN. Regulation of plant translation by upstream open reading frames. Plant Sci. 2014;214:1-12. doi:10.1016/ j.plantsci.2013.09.006

23. Lohmer S, Maddaloni M, Motto M, Salamini F, Thompson RD. Translation of the mRNA of the maize transcriptional activator Opaque-2 is inhibited by upstream open reading frames present in the leader sequence. Plant Cell. 1992;5:65-73. doi:10.1105/tpc.5.1.65.

24. Locatelli F, Magnani E, Vighi C, Lanzanova C, Coraggio I. Inhibitory effect of myb7 uORF on downstream gene expression in homologous (rice) and heterologous (tobacco) systems. Plant Mol Biol. 2002;48:309-18. doi:10.1023/ A:1013340004348.

25. Hanfrey C, Francescetti M, Mayer MJ, Illingworth C, Michael AJ. Abrogation of upstream open reading frame-mediated translational control of a plant S-adenosylmethionine decarboxylase results in polyamine disruption and growth perturbations. J Biol Chem. 2002;277:44131-9. doi:10.1074/ jbc.M206161200.

26. Emebiri L, Michael P, Moody D. Enhanced tolerance to boron toxicity in two-rowed barley by marker-assisted introgression of favourable alleles derived from Sahara 3771. Plant Soil. 2009;314:77-85. doi:10.1007/ s11104-008-9707-0.

27. Schnurbusch T, Hayes J, Sutton T. Boron toxicity tolerance in wheat and barley: Australian perspectives. Breeding Sci. 2010;60:297-304. doi:10.1270/ jsbbs.60.297.

28. Coventry SJ, Smith DS, McKay A, Eckermann PJ, Eglinton JK. Investigating root architecture in barley and responses to salinity and high boron. In: Ceccarelli S, Grando S, editors. Proc $10^{\text {th }}$ Int Barley Genet Symp. Alexandria, Egypt: ICARDA; 2008. pp. 180-189.

29. Moody D. 'SLOOP VIC' Application No. 2002/066. Plant Varieties J. 2003;16:288-92.
30. Burton RA, Shirley NJ, King BJ, Harvey AJ, Fincher GB. The CesA gene family of barley. Quantitative analysis of transcripts reveals two groups of co-expressed genes. Plant Physiol. 2004;134:224-36. doi:10.1104/pp. 103.032904.

31. Hayes JE, Pallotta M, Baumann U, Berger B, Langridge P, Sutton T. Germanium as a tool to dissect boron toxicity effects in barley and wheat. Functional Plant Biol. 2013;40:618-27. doi:10.1071/FP12329.

32. Wheal MS, Fowles TO, Palmer LT. A cost-effective acid digestion method using closed polypropylene tubes for inductively coupled plasma optical emission spectrometry (ICP-OES) analysis of plant essential elements. Anal Methods. 2011;3:2854-63. doi:10.1039/C1AY05430A.

33. Wolf B. Improvements in the azomethine-H method for the determination of boron. Comm Soil Sci Plant Anal. 1974;5:39-44. doi:10.1080/ 00103627409366478

34. Pallotta MA, Warner P, Fox RL, Kuchel H, Jefferies SJ, Langridge P. Marker assisted wheat breeding in the southern region of Australia. In: Pogna NE, editor. Proc $10^{\text {th }}$ Int Wheat Genet Symp. Paestum, Italy: Istituto Sperimentale per la Cerealicoltura; 2003. p. 789-91.

35. Franckowiak JD, Lundqvist U. Coordinator's report: Rules for nomenclature and gene symbolization in barley. Barley Genet Newsletter. 2010;40:178-82.

\section{Submit your next manuscript to BioMed Central and take full advantage of:}

- Convenient online submission

- Thorough peer review

- No space constraints or color figure charges

- Immediate publication on acceptance

- Inclusion in PubMed, CAS, Scopus and Google Scholar

- Research which is freely available for redistribution 